\title{
Symmetries, Singularities and the De-emergence of Space
}

\author{
Thibault Damour ${ }^{1}$ and Hermann Nicolai ${ }^{2}$ \\ ${ }^{1}$ Institut des Hautes Etudes Scientifiques \\ 35, Route de Chartres, F-91440 Bures-sur-Yvette, France \\ ${ }^{2}$ Max-Planck-Institut für Gravitationsphysik \\ Albert-Einstein-Institut \\ Mühlenberg 1, D-14476 Potsdam, Germany \\ Email: damour@ihes.fr, nicolai@aei.mpg.de
}

\begin{abstract}
Recent work has revealed intriguing connections between a Belinsky-Khalatnikov-Lifshitz-type analysis of spacelike singularities in General Relativity and certain infinite dimensional Lie algebras, and in particular the 'maximally extended' hyperbolic Kac-Moody algebra $E_{10}$. In this essay we argue that these results may lead to an entirely new understanding of the (quantum) nature of space(-time) at the Planck scale, and hence - via an effective 'de-emergence' of space near a singularity - to a novel mechanism for achieving background independence in quantum gravity.
\end{abstract}

1. Introduction. A key challenge for a future theory of quantum gravity is the need to explain the fate of space-time singularities, where classical general relativity breaks down, and space and time 'come to an end'. This challenge concerns in particular spacelike (cosmological) singularities, the most prominent example of which is the big bang singularity that gave birth to our universe. At issue here is not only the question of whether and how quantum effects might resolve the singularity, but the very meaning of the term 'singularity resolution' itself. The latter hinges essentially on what the correct theory is, and will almost certainly require new concepts that go beyond established notions of space and time.

A naive extension of quantum mechanics would suggest that singularity resolution works essentially in the same way for quantum general relativity as 
it does for the hydrogen atom. There, as is well known, the expected classical 'collapse' of an electron towards the $1 / r$ singularity of the Coulomb potential is resolved by the Heisenberg uncertainty principle and the quantum mechanical smearing of the electron wave function, which allows the electron to stay in a stable bound state around $r=0$. This mechanism is often invoked in canonical approaches to quantum gravity, where one would thus hope to be able to replace the classical time evolution of the spatial geometry, described as a 'trajectory' in the space of 3-geometries (that is, Wheeler-DeWitt superspace), by a quantum mechanical description in terms of a wave functional which 'smears' the 3-geometries over the singular classical trajectories. This line of thought has been extensively pursued in the simplified context of the mini-superspace approximation, with varying results: while models derived from (or motivated by) loop quantum gravity generally tend to predict a 'bounce' providing a quantum mechanical bridge between two classical universes [1], the more conventional quantum geometrodynamical treatment of the mini-superspace Wheeler-DeWitt equation shows no such evidence [2].

In this essay we would like to outline a very different proposal, motivated by recent work $[3,4]$, where the singularity is 'resolved' by the effective 'disappearance' of space, and the replacement of the dynamical fields, most notably the spatial metric $g_{i j}(t, \mathbf{x})$, by a single dynamical variable $\mathcal{V}(t)$ belonging to an infinite dimensional coset space and depending only on time. Our proposal is based on the discovery of a profound relation between an analysis à la Belinsky-Khalatnikov-Lifshitz (BKL) of spacelike singularities $[5,6]$ on the one hand, and the theory of indefinite Kac-Moody algebras on the other [7, 8] (see [9] for an introduction to the theory of Kac-Moody algebras). More specifically, the main conjecture of [3], formulated in the context of the maximally extended $D=11$ supergravity [10], relates a BKL-type expansion in spatial gradients at a given spatial point to a Lie algebraic expansion in the height of certain roots of the 'maximally extended' hyperbolic Kac-Moody algebra $E_{10}$. Thereby the time evolution of 10-dimensional geometric data is mapped onto an effectively one-dimensional dynamics, namely 
a (constrained) null geodesic motion in the infinite dimensional coset space $E_{10} / K\left(E_{10}\right)$, which is formally defined as the quotient of the group $E_{10}$ by its maximal compact subgroup $K\left(E_{10}\right)^{1}$. The appearance of $E_{10}$ in this context is both unexpected and remarkable, because $E_{10}$ enjoys a similarly distinguished status among the infinite dimensional Lie groups ${ }^{2}$ as the exceptional group $E_{8}$ does in the Cartan-Killing classification of the finite dimensional simple Lie groups [13].

2. BKL and cosmobilliards. We start by summarizing the BKL-type analysis of the near spacelike singularity limit, that is, of the asymptotic behaviour of various fields, and in particular the (spatial) metric, near a singular hypersurface, here taken to be 'located' at $T=0$ in proper time $T$. To this aim, it is convenient [8] to decompose the $D=11$ metric $g_{\mu \nu}$ into non-dynamical and dynamical components, namely the lapse $N$ and the shift vector $N^{m}$ (set here to zero), and into 'diagonal' and 'off-diagonal' components $e^{-2 \beta^{a}}$ and $\theta^{a}$, respectively, such that the line element becomes

$$
d s^{2}=-N^{2} d t^{2}+\sum_{a=1}^{10} e^{-2 \beta^{a}} \theta^{a}{ }_{i} \theta_{j}^{a} d x^{i} d x^{j}
$$

Here, the 'off diagonal' components $\theta^{a}{ }_{i}$ entering the Iwasawa decomposition of the spatial metric $g_{i j}$ are upper triangular matrices with 1's on the diagonal. We choose a gauge for $N$ in terms of $g_{i j}$ in such a way that $N \sim \mathcal{O}(T) \rightarrow 0$ when $T \rightarrow 0$. Thus $t \sim-\log T$ becomes a 'Zeno-like' time coordinate with $t \rightarrow+\infty$ as $T \rightarrow 0$.

The Hamiltonian constraint, at a given spatial point, can be written as

$\mathcal{H}\left(\beta^{a}, \pi_{a}, Q, P\right)=\tilde{N}\left[\frac{1}{2} G^{a b} \pi_{a} \pi_{b}+\sum_{A} c_{A}(Q, P, \partial \beta, \partial Q) \exp \left(-2 w_{A}(\beta)\right)\right]$

\footnotetext{
${ }^{1}$ The emergence of $E_{10}$ in the dimensional reduction of maximal supergravity to one dimension had been conjectured already long ago [11]. See also [12] for a conceptually very different proposal based on $E_{11}$.

${ }^{2}$ For simplicity of notation, we denote the group and its Lie algebra by the same symbol.
} 
with the rescaled lapse $\tilde{N} \equiv N / \sqrt{g}$, where $g$ is the determinant of the spatial metric. Here $\pi_{a}$ (with $a=1, \ldots, 10$ ) are the canonical momenta conjugate to the logarithmic scale factors $\beta^{a}$, and $G^{a b}$ is the (Lorentzian) DeWitt 'superspace' metric induced by the Einstein-Hilbert action. $(Q, P)$ denote the remaining canonical degrees of freedom associated to the off-diagonal metric components $\theta^{a}{ }_{i}$ and various matter degrees of freedom [such as the 3 -form $A_{\mu \nu \lambda}(t, \mathbf{x})$ of $D=11$ supergravity], as well as their respective conjugate momenta, and $(\partial \beta, \partial Q)$ are the spatial gradients of $\beta$ and $Q$. The exponential terms in (2) involve linear forms $w_{A}(\beta) \equiv w_{A a} \beta^{a}$, where the specific coefficients $w_{A a}$ and the range of labels $A$ depend on the model under consideration (see [8] for details).

The BKL limit $T \rightarrow 0$ amounts to considering the large $\beta$ limit in Eq. (2), and is determined by the exponential 'walls' $\propto \exp \left(-2 w_{A}(\beta)\right)$ [8]. The latter can be ordered in 'layers'. The first layer, corresponding to the subset of 'dominant walls' $w_{A^{\prime}}(\beta)$ — whose coefficients $c_{A^{\prime}}(Q, P, \partial \beta, \partial Q)$ can be shown to be non-negative - confine the motion in $\beta$-space to a fundamental billiard chamber defined by the inequalities $w_{A^{\prime}}(\beta) \geq 0$. The remaining (subdominant) exponential walls introduce fractional corrections to the chaotic motion of $\left(\beta^{a}, \pi_{a}\right)$ within the fundamental billiard chamber. All the other dynamical variables $(Q, P)$, together with their spatial gradients, 'freeze' as $T \rightarrow 0$, and thus exhibit a very different behavior in this limit.

3. Coset space dynamics. Let us next consider an a priori very different dynamical system, namely null geodesic motion on the infinite-dimensional coset space $E_{10} / K\left(E_{10}\right)$. A curve on this coset space can be parametrized by a time-dependent (but space independent) element of the $E_{10}$ group in upper triangular (Iwasawa) form: $\mathcal{V}(t)=\exp h(t) \exp \nu(t)$. Here, $h(t)=\beta^{a}(t) H_{a}$ belongs to the 10-dimensional Cartan subalgebra (=CSA) of $E_{10}$. Our use of the same notation as above is justified by the fact that we will eventually identify the ten CSA coordinates $\beta^{a}$ of $E_{10}$ with the logarithms of the ten 'diagonal' components of the spatial metric $g_{i j}$ introduced above. On the other hand, $\nu(t)=\sum_{\alpha>0} \nu^{\alpha}(t) E_{\alpha}$ belongs to a (Borel) subalgebra of $E_{10}$ and 
has an infinite number of components labelled by positive roots $\alpha$ of $E_{10}$. The geodesic action is formally very simple; it reads

$$
\int d t \mathcal{L}(t)=\int \frac{d t}{n(t)}\langle\mathcal{P}(t) \mid \mathcal{P}(t)\rangle
$$

where $\mathcal{P}:=(1 / 2)\left[\dot{\mathcal{V}} \mathcal{V}^{-1}+\left(\dot{\mathcal{V}} \mathcal{V}^{-1}\right)^{T}\right]$ is the "velocity'3, $\langle. \mid$.$\rangle is the standard in-$ variant bilinear form generalizing the finite dimensional matrix trace [9], and $n(t)$ is a one-dimensional 'lapse' needed to ensure (time) reparametrisation invariance of the action (3). The Zeno-like coordinate time $t$ of the previous section is recovered upon identifying $n$ with the rescaled lapse $\tilde{N}$ introduced after (2) and choosing the gauge $n(t)=1$.

Varying (3) w.r.t. to the lapse $n$, we obtain the Hamiltonian constraint:

$H\left(\beta^{a}, \pi_{a}, \nu, p\right)=n\left[\frac{1}{2} G^{a b} \pi_{a} \pi_{b}+\sum_{\alpha>0} \sum_{s=1}^{\operatorname{mult}(\alpha)}\left(\Pi_{\alpha, s}\left(\nu^{\alpha}, p_{\alpha}\right)\right)^{2} \exp (-2 \alpha(\beta))\right]$

where $\pi_{a}$ denote the conjugate momenta of the ten diagonal CSA coordinates $\beta^{a}$, and $p_{\alpha}$ denote the conjugate momenta of the 'off-diagonal' coordinates $\nu^{\alpha}$ parametrizing the Borel part of $\mathcal{V}$, on which the $\Pi_{\alpha, s}$ depend [8]. The sum on the r.h.s. of (4) ranges over all positive roots $\alpha$ of $E_{10}$ with their multiplicities $[=\operatorname{mult}(\alpha)]$. We recall that the roots $\alpha$ are linear forms on the CSA, that is, we have $\alpha(\beta) \equiv \alpha_{a} \beta^{a}$ for the exponents in (4). Although the dynamics encapsulated in (4) is very complicated, a general feature is that in order to satisfy $H=0$, we must always have $G^{a b} \pi_{a} \pi_{b} \leq 0$; this means that the coset null geodesics must always maintain a future-directed CSA velocity $\pi^{a}$, hence cannot bounce backwards in $\beta$ space.

4. Correspondence between BKL and coset-space dynamics. The formal similarity between the gravitational Hamiltonian (2) (considered at a given spatial point) and the coset Hamiltonian (4) is evident, but the precise correspondence has so far been established only for a limited number of

\footnotetext{
${ }^{3}$ Here the transpose ${ }^{T}$ denotes the negative of the Chevalley ('compact') involution [9]. The compact subalgebra $K\left(E_{10}\right)$ is thus spanned by the 'antisymmetric' elements of $E_{10}$.
} 
terms. In particular, the metric $G^{a b}$ entering (4), which is the restriction of the invariant bilinear form on $E_{10}$ to its CSA, happens to be identical with the DeWitt metric appearing in (2). This fact enables us to identify the space of logarithmic scale factors with the Cartan subalgebra of $E_{10}$ (as anticipated by our notation). Moreover, one can analyze the asymptotic dynamics of the coset variables $\left(\beta^{a}, \pi_{a}, \nu^{\alpha}, p_{\alpha}\right)$ in the limit of large $\beta$ 's. At first order in an expansion in 'height' of the simple roots of $E_{10}$, one finds that the CSA variables $\beta$ are confined to a chaotic billiard motion within the Weyl chamber of $E_{10}$. The latter is defined by the inequalities $\alpha_{i}(\beta) \geq 0$, where the $\alpha_{i}$ 's $(i=1, \cdots, 10)$ are the simple roots of $E_{10}$, and turns out to coincide with the fundamental BKL billiard chamber defined by the dominant potential walls $w_{A^{\prime}}(\beta)$ for $D=11$ supergravity. Consideration of the subleading exponential walls in both models now shows that one can actually identify the two dynamics up to height 30, i.e. much beyond the leading billiard dynamics (corresponding to height one only) [3]. This result suggests the existence of a hidden equivalence between the two models, i.e. the existence of a map preserving the dynamics between the infinite tower of coset variables $\left(\beta^{a}, \pi_{a}, \nu^{\alpha}, p_{\alpha}\right)$, and the infinite sequence of spatial Taylor coefficients $\left(\beta(\mathbf{x}), \pi(\mathbf{x}), Q(\mathbf{x}), P(\mathbf{x}), \partial \beta(\mathbf{x}), \partial Q(\mathbf{x}), \partial^{2} \beta(\mathbf{x}), \partial^{2} Q(\mathbf{x}), \ldots\right)$ formally describing the dynamics of the (super)gravity fields $(\beta(\mathbf{x}), \pi(\mathbf{x}), Q(\mathbf{x}), P(\mathbf{x}))$ in the neighborhood of some given spatial point $\mathbf{x}$. In this way, a skeletonization of the (super-)gravity fields by means of their infinite towers of spatial gradients gets related to a purely Lie algebraic expansion in terms of heights of roots. While the full details of this correspondence (which is expected to be ultimately very non-local in the space-time fields) remain to be worked out, it has been possible recently to extend these results also to the fermionic sector on both sides $[14,15]$.

Most importantly for our present proposal, certain (partially) known quantum corrections to the classical supergravity action can be shown to be compatible with specific terms, of very large height, present in the coset 
action [4]. For instance, the leading term quartic in the Weyl curvature,

$$
\begin{aligned}
\mathcal{L}^{(4)}=192 N & \sqrt{g}\left(-C^{A B C D} C_{A B}{ }^{E F} C_{C E}{ }^{G H} C_{D F G H}\right. \\
& \left.+4 C^{A B C D} C_{A}{ }^{E} C^{F} C_{E}{ }^{G}{ }_{B}{ }^{H} C_{F G D H}\right)
\end{aligned}
$$

is dominated, near the singularity, by an exponential term $\propto \exp [-2 \alpha(\beta)]$ in the coset Hamiltonian (4) for a specific imaginary $E_{10}$ root $\alpha$ of height (minus) 115. Detailed study of the combination of curvature terms in (5) has established an inequality [4] confirming the no bounce property (in $\beta$ space) exhibited by the coset dynamics, and briefly explained after (4).

5. The cosmological singularity: a new paradigm? The evidence summarized above suggests an entirely new picture of the (quantum) fate of space and time at a cosmological singularity. Namely we here propose to take seriously the idea that near the singularity (i.e. when the curvature gets larger than the Planck scale) the description in terms of a spatial continuum and space-time based (quantum) field theory breaks down, and should be replaced by a much more abstract Lie algebraic description. Thereby the information previously encoded in the spatial variation of the geometry and of the matter fields gets transferred to an infinite tower of Lie algebraic variables depending only on 'time'. In other words, we are led to the conclusion that space - and thus, upon quantization, also space-time - actually disappears (or 'de-emerges') as the singularity is approached. ${ }^{4}$ There is no 'quantum bounce' bridging the gap between an incoming collapsing and an outgoing expanding quasi-classical universe. Instead 'life continues' at the singularity for an infinite affine time, however, with the understanding that (i) dynamics no longer 'takes place' in space, and (ii) the infinite affine time interval [measured, say, by the Zeno-like time coordinate $t$ of (3)] corresponds to a sub-Planckian interval $0<T<T_{\text {Planck }}$ of geometrical proper time.

\footnotetext{
${ }^{4}$ We have in mind here a 'big crunch', i.e. we conventionally consider that we move towards the singularity. Mutatis mutandis, we would say that space 'appears' or 'emerges' at a big bang.
} 
Upon quantization, the geodesic equations of motion following from (3) are replaced by a quantum version of the Hamiltonian constraint (4), analogous to the Wheeler-DeWitt equation, and acting on some 'wave function of the coset particle' $\Psi=\Psi\left(\beta^{a}, \nu^{\alpha}\right)$ depending on the coset variables. This, then, is the step where time also 'disappears': as in all canonical approaches to quantum gravity, the wave function (or functional) $\Psi$ no longer depends on any extrinsic 'time' [although one can, of course, choose a 'clock field' among the coset variables so as to define an 'operational' time, in terms of which the quantum dynamics of the remaining variables can be parametrized]. The quantum constraint would take the form of a Klein-Gordon-like equation ${ }^{5}$

$$
\square \Psi\left(\beta^{a}, \nu^{\alpha}\right)=0
$$

where $\square$ is the (formal) Laplace-Beltrami operator on the infinite-dimensional (Lorentzian) coset manifold $E_{10} / K\left(E_{10}\right)$. It is noteworthy that all reference to space and time has disappeared in (6). The discretization of finitedimensional duality symmetries upon quantization, well known from toroidal compactification in string theory, would then suggest that the "wave function of the universe' is a modular form over the arithmetic group $E_{10}(\mathbb{Z})[16]$.

6. Outlook. If correct, the picture outlined here will not only affect our understanding of what 'happens' at a cosmological singularity, but may also shed a completely new light on the issue of background independence in quantum gravity. More succinctly, taking the quantum coset dynamics (6) as a guiding principle, the correct theory of quantum gravity may well turn out to be background independent in the sense that near the singularity, the theory - rather than 'quantizing' the spatial geometry, or some other spatially extended background structure - simply does away with the background altogether, whence the whole issue would become moot!

Let us also note some potentially important implications of this picture for the so-called 'information loss paradox' in black hole physics. Indeed, the present ideas might also be applied to the case of a 'localized' big crunch

\footnotetext{
${ }^{5}$ Or a 'Dirac-like' (first order) constraint if fermions are included [14, 15].
} 
(as the one inside a black hole formed within an asymptotically flat spacetime). It would then suggest that some of the information contained within the horizon might transmigrate to the state of motion of a coset 'baby particle' (whose dynamics describes physics at sub-Planckian scales near the big crunch). The Hawking evaporation of the black hole containing this localized big crunch poses interesting conceptual challenges with regard to an infinite affine 'coset life-time' near the singularity.

It is well known that symmetry concepts have been of central importance in the advancement of theoretical physics over the last century. They have been a key ingredient in the development of the two most successful theories of physics, namely general relativity (via the principle of general covariance) and the standard model of elementary particle physics (via gauge invariance and Yang Mills quantum field theories). In view of its distinguished place among all Lie algebras, $E_{10}$ is a most worthy candidate for symmetry of nature, deeply intertwining space-time with matter degrees of freedom, and thus necessarily implying a unification of gravity and matter. For this reason, we can anticipate for it a key role in elucidating the quantum nature of spacetime, and hence space-time singularities.

Acknowledgements: It is a pleasure to thank our collaborators Marc Henneaux and Axel Kleinschmidt for many fruitful discussions over the years. H.N. would also like to thank KITP, Santa Barbara, for hospitality in January 2007, and the workshop participants for discussions and inspiration.

\section{References}

[1] M. Bojowald, Singularities and quantum gravity, gr-qc/0702144

[2] C. Kiefer, Quantum gravity, Clarendon Press, 2004

[3] T. Damour, M. Henneaux and H. Nicolai, $E_{10}$ and a "small tension expansion" of M-theory, Phys. Rev. Lett. 89 (2002) 221601 
[4] T. Damour and H. Nicolai, Higher order $M$ theory corrections and the Kac-Moody algebra $E_{10}$, Class. Quant. Grav. 22 (2005) 2849

[5] V. A. Belinsky, I. M. Khalatnikov and E. M. Lifshitz, Oscillatory Approach To A Singular Point In The Relativistic Cosmology, Adv. Phys. 19 (1970) 525

[6] C. W. Misner, Quantum Cosmology. 1, Phys. Rev. 186, 1319 (1969)

[7] T. Damour and M. Henneaux, $E_{10}, B E_{10}$ and arithmetical chaos in superstring cosmology, Phys. Rev. Lett. 86 (2001) 4749

[8] T. Damour, M. Henneaux and H. Nicolai, Cosmological Billiards, Class. Quant. Grav. 20 (2003) R145-R200

[9] V. G. Kac, Infinite dimensional Lie algebras, 3rd edition, Cambridge University Press (Cambridge, 1990)

[10] E. Cremmer, B. Julia and J. Scherk, Supergravity theory in 11 dimensions, Phys. Lett. B 76 (1978) 409-412

[11] B. Julia, in: Lectures in Applied Mathematics, Vol. 21 (1985), AMSSIAM, p. 335; preprint LPTENS 80/16

[12] P. C. West, E(11) and M theory, Class. Quant. Grav. 18, 4443 (2001)

[13] See e.g. http://aimath.org/E8

[14] T. Damour, A. Kleinschmidt and H. Nicolai, Hidden symmetries and the fermionic sector of eleven-dimensional supergravity, Phys. Lett. B 634, 319 (2006); $K(E(10))$, supergravity and fermions, JHEP 0608, 046 (2006)

[15] S. de Buyl, M. Henneaux and L. Paulot, Extended E(8) invariance of 11-dimensional supergravity, JHEP 0602, 056 (2006)

[16] O. J. Ganor, Two conjectures on gauge theories, gravity, and infinite dimensional Kac-Moody groups, J. Brown, O. J. Ganor and C. Helfgott, $M$-theory and E(10): Billiards, branes, and imaginary roots, JHEP 0408, 063 (2004) 